\title{
Construcción del hábitat en la periferia de la Ciudad de México. Estudio de caso en Zumpango
}

Fermin Cruz-Muñoz. Instituto Politécnico Nacional, Ciudad de México, México. Georgina Isunza. Instituto Politécnico Nacional, Ciudad de México, México.

RESUMEN | Este trabajo contribuye a la discusión de la problemática socioespacial implicada en la construcción del hábitat en la periferia de la Ciudad de México. Desde la perspectiva del nuevo modelo de expansión urbana, se identifican las deficiencias de habitabilidad en los conjuntos habitacionales periféricos. Ellas han sido documentadas en el estudio de caso de Zumpango, municipio periurbano que experimenta la transformación del hábitat desde un entorno rururbano popular, al conformado por núcleos de población residente en grandes desarrollos inmobiliarios de vivienda que no logran convertirse en espacios habitables, lo que ocasiona el grave problema del abandono de la vivienda nueva. Para evaluar la calidad del hábitat, se elabora una propuesta propia con base en cuatro indicadores: (i) la infraestructura básica, como agua potable, drenaje, alcantarillado, energía eléctrica y alumbrado público; (ii) el equipamiento básico, como escuelas y centros de salud; (iii) la accesibilidad urbana, tanto local como metropolitana; y (iv) la existencia de espacios públicos de convivencia, materializados en parques, jardines o plazas.

PALABRAS CLAVE | urbanización, periferia urbana, mercado inmobiliario.

ABSTRACT | This paper contributes to the discussion of socio-spatial problems involved in the construction of the habitat in the periphery of Mexico City. From the perspective of the New Urban Expansion Model, habitability deficiencies are identified and documented on the findings of a study case. Zumpango is a periurban municipality that experiences habitat transformations from popular rururban environment to large housing estate development centers that fail to create living spaces, causing numerous cases of new housing abandonment. In order to evaluate habitat quality, a proposal based on four indicators is elaborated: (i) Elementary infrastructure such as drinking water, drainage, sewerage, electricity and public lighting; (ii) The basic urban equipment as schools and health centers; (iii) Local and metropolitan urban accessibility, and (iv) The existence of convivial public spaces, expressed in parks, public gardens and plazas.

KEY WORDS | urbanization, urban periphery, real estate market. 


\section{Introducción}

En México se reconocen cinco millones de viviendas deshabitadas, que representan 15\% del parque habitacional (Instituto Nacional de Estadística y Geografía [INEGI], 2010), lo cual convierte el abandono de vivienda en una preocupación para gobiernos nacionales, subnacionales y locales, así como para las empresas inmobiliarias, que reclaman altas tasas de retorno de sus inversiones.

Bajo la mirada de la investigación académica, este fenómeno se ubica en el marco de las mutaciones que experimentan numerosas metrópolis latinoamericanas. Si bien el tema del abandono de vivienda es complejo y atiende a diversas causas dependiendo de las configuraciones socioterritoriales prevalecientes, en el caso de la Zona Metropolitana de la Ciudad de México denota un grave problema de habitabilidad (Isunza, 2015).

El objetivo de este trabajo es contribuir a la comprensión de los obstáculos que enfrenta el proceso de construcción del hábitat en la periferia metropolitana de la Ciudad de México ante un contexto de oferta inmobiliaria de masivos conjuntos habitacionales. La calidad del hábitat se analizará a partir de cuatro categorías propuestas: (i) la infraestructura básica, como agua potable, drenaje, alcantarillado, energía eléctrica y alumbrado público; (ii) el equipamiento básico, como los centros educativos, de salud y abasto; (iii) la accesibilidad urbana, tanto local como metropolitana; y (iv) la existencia de espacios públicos de convivencia, materializados en parques, jardines o plazas. Estos elementos construidos le otorgan al hábitat las cualidades necesarias para aquella convivencia social que motiva la cohesión de la población y posibilita un proceso de apropiación del espacio, por lo que su deficiencia motivaría a los residentes a modificar su entorno o buscar hábitats más adecuados para su desarrollo.

Según la hipótesis que subyace en este trabajo, la nueva expansión urbana basada en la construcción de conjuntos habitacionales localizados en la periferia urbana de la metrópoli, no genera condiciones adecuadas para la construcción social del hábitat, toda vez que se construye vivienda, pero escasamente se generan las condiciones de su entorno que le imprimen habitabilidad.

Un espacio habitable permite que la vida cotidiana se organice dentro de un espacio de vida polifuncional próximo al hogar; sin embargo, hoy se ha pasado a un modelo de vida donde las diferentes actividades se hallan dispersas en una extensa trama urbana, de tal suerte que la vida cotidiana no se organiza en el entorno local (espacio de proximidad), sino a lo largo de un espacio metropolitano (Avellaneda \& Lazo, 2011, p. 55). Así, pese a la incorporación de algunos servicios en los conjuntos habitacionales, el hábitat de la nueva periferia urbana tiene una calidad deficiente, minando con ello la capacidad de adecuación del espacio construido y dificultando la construcción del espacio de vida.

La investigación se documenta en un estudio de caso en Zumpango, municipio localizado en el área metropolitana de la Ciudad de México (АмСм) en el que se observa la transformación del hábitat, que ha pasado de ser un espacio predominantemente rururbano popular, a uno conformado por fraccionamientos ${ }^{1}$ construidos 
por las empresas inmobiliarias. En este entorno los habitantes enfrentan severos problemas relacionados con la habitabilidad, situación que en numerosos casos termina con el abandono de la vivienda y del hábitat correspondiente.

\section{Estrategia metodológica para el análisis de la construcción del hábitat}

La habitabilidad es un concepto polisémico, pero en su nuevo enfoque ha rebasado el ámbito de la vivienda, para incorporar los satisfactores que ofrece el entorno a las personas; en este caso, es entendida como la condición material, ambiental y cultural del espacio habitado por las personas, ligada a la valoración del entorno de los habitantes y que ofrece satisfacción en términos de seguridad, identidad, comodidad y apropiación (Tarchópulos \& Ceballos, 2003, p. 17).

De acuerdo con Zulaica y Celemín (2008), la habitabilidad se refiere a "la capacidad de los espacios construidos para satisfacer las necesidades objetivas y subjetivas de los individuos y grupos" (p. 130). Por lo tanto, expresa una aspiración de bienestar que involucra, además de las condiciones físicas de la vivienda, el ambiente sociocultural (prácticas sociales cotidianas, pautas de consumo, la identidad y el imaginario colectivo, lazos sociales) que contribuye al bienestar personal y colectivo que proporciona el hecho de residir en un asentamiento satisfactorio determinado (p. 131).

Una mirada a los entornos locales más dispersos, alejados de las áreas que ejercen la mayor centralidad urbana, en donde numerosas familias pueden acceder a la vivienda, nos conduce a pensar en la habitabilidad como una demanda social de disponibilidad de las condiciones requeridas para satisfacer las necesidades socialmente reconocidas (Arcas-Abella, Pagès-Ramon \& Casals-Tres, 2011, p. 66).

Esta investigación profundiza en el hábitat construido en torno a la vivienda en una periferia urbana lejana, caracterizada esencialmente por la escasa articulación territorial de sus habitantes y un creciente número de personas que realizan viajes, principalmente por motivos de trabajo, a los centros de concentración de empleo en las zonas centrales e intermedias de la metrópoli. De esta forma, la construcción de un entorno residencial con los medios de consumo suficientes adquiere vital importancia para dotar de los atributos de habitabilidad a esos espacios periféricos.

Las investigaciones que buscan operacionalizar el estudio del hábitat a partir de determinadas variables son muy limitadas. Por un lado, es posible identificar trabajos que acotan el análisis a las condiciones de la vivienda (Hernández \& Velázquez, 2014; Mac Donald, 2004), respondiendo a la concepción de hábitat enfocada solamente en las cualidades de las edificaciones destinadas a la residencia. Por otro lado, algunos estudios construyen aproximaciones sobre los componentes que le otorgan al hábitat urbano las cualidades necesarias que beneficien el habitar y, por ende, la habitabilidad. Romero y Mesías (2004, p. 29) reconocen como componentes de la habitabilidad la amplitud territorial del hábitat, y mencionan al respecto elementos como la infraestructura de servicios, y otros que favorecen la construcción de relaciones, como son las redes viales y de transporte público que facilitan la movilidad de la población, así como los espacios de convivencia donde se construyen y desenvuelven las relaciones sociales. También mencionan los espacios de trabajo, educación y esparcimiento como aspectos que constituyen al 
hábitat. Por su parte, Brites (2012) analiza el hábitat de una población relocalizada hacia la nueva periferia urbana, y aunque no identifica claramente sus categorías de análisis, menciona como factores que definen la deficiencia del hábitat, la ausencia de servicios e infraestructura.

Una aproximación más estructurada es la expuesta por Hurtado y Villagómez (1992, p. 37), quienes definen las necesidades básicas de una población urbana para lograr una adecuada condición de vida. La obtención de alimento, vestido y otros bienes necesarios para el correcto funcionamiento de la vivienda les otorga vital importancia a los centros de comercialización de bienes básicos de consumo. Destacan también la importancia de servicios como educación y salud, los cuales son proveídos por los elementos de equipamiento. Por último, subrayan la necesidad de dotación de redes de infraestructura, como la de agua potable, saneamiento y transporte público, pues son fundamentales para el correcto desenvolvimiento de las actividades en los espacios tanto urbanos como arquitectónicos.

A partir de las reflexiones anteriores, en este trabajo se elabora una propuesta propia destinada a evaluar la calidad del hábitat de los espacios y servicios públicos en el caso de estudio, a partir de cuatro categorías:

a. Las redes de infraestructura básica dotada por el gobierno local, a saber: el agua potable, la red de drenaje, alcantarillado, de energía eléctrica y alumbrado público. Todos estos componentes son fundamentales para la realización de las actividades domésticas, ejecutadas esencialmente en torno a la vivienda, y para las actividades colectivas manifiestas en los espacios públicos y servicios de equipamiento.

b. Elementos básicos de equipamiento, esencialmente la educación, la salud y el abasto de bienes básicos de consumo, los cuales potencializan en gran medida la calidad de vida de la población.

c. La accesibilidad espacial que ofrece el hábitat local, que permite la movilidad hacia los otros espacios de vida, como los de trabajo. La accesibilidad se construye a partir de la traza de las vías de comunicación, como las vialidades, y de los servicios de transporte públicos.

d. Los espacios abiertos públicos de convivencia, que representan los puntos de reunión que coadyuvan a la construcción de una pertenencia e identidad.

Estos cuatro elementos sustentan las bases para la construcción de un hábitat de calidad, pues otorgan el sustrato material para el habitar. Si bien existe el proceso de apropiación y adecuación del territorio por los habitantes urbanos, es difícil construir un hábitat sin esos cuatro componentes fundamentales en un entorno residencial.

Una fuente de información utilizada para comprender los procesos de construcción del hábitat es la Encuesta "Movilidad residencial en Tecámac y Zumpango, Estado de México", ${ }^{2}$ dirigida a jefes de familia residentes de los conjuntos habitacionales en ambos municipios, con un muestreo estratificado por asignación

2 Véase G. Isunza (2013), "Encuesta Movilidad Residencial en Tecámac y Zumpango, Estado de México", Informe del Proyecto Desarrollos Inmobiliarios y movilidad residencial en la ZMvM, registro 1470, Instituto Politécnico Nacional, octubre de 2013. http://repositoriodigital.ipn.mx/ handle/123456789/16878 
proporcional en los conjuntos habitacionales identificados en cada municipio. Para determinar la muestra se empleó el siguiente procedimiento:

a. Se llevó a cabo una selección por conglomerados de cada uno de los conjuntos.

b. Una vez seleccionados, se revisó la pertinencia en cuanto a costo y distancia de los conjuntos seleccionados.

c. Para conocer el número de encuestas, estas se distribuyeron en función del número de viviendas por conjunto.

Con un error de 5\%, se aplicaron 423 cuestionarios en Tecámac y 419 en Zumpango, utilizando la fórmula de población finita. Este trabajo solo considera los resultados para Zumpango.

\section{Proceso de expansión urbana y construcción del hábitat}

La mayor parte de las ciudades latinoamericanas que actualmente se perfilan como nodos de la red mundial de ciudades (Buenos Aires, Bogotá, Caracas, São Paulo, Rio de Janeiro, Santiago, Lima y la Ciudad de México) han tenido un papel preponderante en la integración económico-territorial. Uno de los principales rasgos que adquieren las metrópolis latinoamericanas en la fase actual de desarrollo capitalista es el fortalecimiento de la tendencia al derrame territorial, caracterizado por el desborde de los límites urbanos preexistentes y la incorporación de localidades rururbanas, dejando a su paso vacíos o semivacíos (De Mattos, 2015, p. 82).

El auge de los desarrollos inmobiliarios contribuye a fortalecer la formación de un periurbano difuso, de baja densidad, motivado por el cambio de residencia de numerosas familias que solo tienen acceso a la vivienda en los espacios periurbanos más lejanos, dando lugar a un patrón de metropolización expandida que agudiza los procesos de polarización y la segregación en la estructura metropolitana. De ahí derivan múltiples deficiencias en la calidad del hábitat.

La expansión urbana en las metrópolis mexicanas también se caracteriza por una tendencia a la dispersión, debido a que se dirige hacia municipios cada vez más lejanos, con importantes rezagos productivos y sociales, muchos de ellos con configuraciones rurales. Sus residentes, productores agropecuarios al borde de la ruina, ofrecen en venta amplias extensiones de suelo a las empresas inmobiliarias para la construcción de grandes conjuntos habitacionales, lo que configura un patrón de urbanización segregado que trastoca la habitabilidad.

El boom inmobiliario es un elemento decisivo para explicar los problemas relacionados con la construcción social del hábitat, ya que ha producido un desfase entre el lugar donde se adquiere la vivienda (ligado al acceso al suelo) y el lugar de trabajo, que generalmente se mantiene cuando las familias cambian de residencia. A ello se suman factores como la localización concentrada de la actividad económica y, en consecuencia, de los puestos de trabajo; también la segmentación laboral plasmada en el territorio; el incremento y la fragmentación del tiempo; el costo y esfuerzo implicados en el desplazamiento, más los graves efectos ambientales que ello implica. 
El fomento a la participación del sector inmobiliario en la dotación de la vivienda a partir de grandes conjuntos habitacionales modificó la tradicional tendencia de expansión urbana. Las empresas inmobiliarias fungen como creadoras de nuevas áreas urbanas, cuya lógica responde esencialmente al mercado inmobiliario y a la generación de utilidades.

Ante esta nueva dinámica, el papel del gobierno se ha limitado a conciliar los posibles problemas de interés público. La planeación urbana ha dejado de ser la principal orientadora del desarrollo territorial. Ello abre las puertas al "nuevo urbanismo”, enfocado en la realización de proyectos sectoriales puntuales en determinadas áreas de las urbes. Esta nueva línea de acción abre posibilidades a las empresas inmobiliarias para la urbanización de las periferias. La expansión urbana deja de ser determinada por las reservas territoriales, que eran concebidas en función de las cualidades favorables a la habitabilidad, para estar ahora dirigida por la lógica del mercado de suelo, donde la ciudad tiende a crecer hacia áreas pertinentes para la rentabilidad de los proyectos inmobiliarios. Se trata de un modelo que responde a "la modificación de las reglas del juego que encuadraban las decisiones y acciones de dos de los actores que mayor incidencia tienen en la producción de la ciudad: empresarios inmobiliarios y familias. Este cambio se deriva del hecho de que la liberalización económica y la desregulación se orientaron explícitamente a remover un conjunto de regulaciones que hasta entonces habían intentado obstaculizar la continuidad de la urbanización estrictamente capitalista” (De Mattos, 2015, p. 83). Ello permite el protagonismo de los mercados inmobiliarios sin mayor intervención de las instituciones públicas para adecuar las estructuras urbanas en función de las nuevas dinámicas sociales, políticas y económicas. Por lo tanto, los marcos jurídicos de planeación se debilitan, dado que no disponen de instrumentos de gestión que favorezcan la construcción social del hábitat. Las periferias urbanas se convierten en hábitats de pobreza cuyos habitantes no logran acceso a los beneficios de la ciudad, dada la deficiencia de las condiciones materiales de infraestructura y equipamiento.

Bajo la perspectiva neoliberal el hábitat también es reinterpretado, entendiéndoselo como el "subsistema de oferta" de vivienda, recursos y servicios urbanos que en teoría están disponibles para la sociedad urbana. Su contraparte es el habitar, que se comprende como el "subsistema de demandas" de vivienda, recursos y servicios urbanos indispensables para el desarrollo de la vida cotidiana de la población (Zulaica \& Celemín, 2008, p.132). En este enfoque, un espacio habitable se interpreta esencialmente como aquel capaz de satisfacer las demandas sociales de cobijo desde la perspectiva del consumo. De esta forma, se asocia la habitabilidad a la satisfacción de necesidades mediante el consumo (Arcas-Abella et al., 2011, p. 84). Por lo tanto, en los actuales procesos de urbanización y de construcción de los espacios de vida, el hábitat está determinado por la oferta de bienes, servicios y condiciones que pudiera llegar a abastecer el sector privado (Zulaica \& Celemín, 2008, p. 135). El papel de las instituciones gubernamentales queda limitado a la dotación del equipamiento y los espacios de convivencia necesarios para la construcción de un hábitat adecuado a la realización de prácticas sociales, que ahora se circunscriben a ejercicios de consumo.

En general, los gobiernos municipales enfrentan grandes desafíos para proporcionar servicios tanto a la nueva población, que proviene principalmente de las 
áreas centrales e intermedias de la Ciudad de México, como a los viejos residentes, originando un desfase entre las competencias de gobierno en el nivel local, con las nuevas, crecientes y diversificadas necesidades de la población. Adicionalmente, los gobiernos municipales son incapaces de promover arreglos institucionales entre los distintos niveles de gobierno que confluyen en el espacio metropolitano, poniendo en evidencia sus escasas posibilidades de enfrentar los efectos más perniciosos de esta modalidad de expansión urbana (Isunza \& Méndez, 2011, p. 109). De esta forma, a las deficiencias del hábitat de la urbanización informal por la ausencia de un diseño urbano que incluya todos los servicios básicos, bajo la nueva lógica de urbanización se suman las deficiencias de la vivienda formalmente construida, que se caracterizan por una escasez o una disfuncionalidad de aquellos espacios públicos que permiten tanto la apropiación social del territorio como la construcción de un hábitat propio. Se mantiene la condición de que al vivir en un barrio o conjunto habitacional precario, se reduce el acceso y las oportunidades de empleo, educación y servicios, solo que ahora bajo la dinámica del libre mercado, donde las empresas no consideran a este segmento de la población como mercado potencial para ofrecerle servicios de equipamiento.

Sin el apoyo técnico y político, y a pesar de la oportunidad que representa la inversión privada en la construcción de la ciudad, lo más probable es que continúe la multiplicación de asentamientos precarios en las metrópolis latinoamericanas, construyéndose hábitats carentes de condiciones adecuadas para la convivencia comunitaria y el desarrollo de las actividades cotidianas. Es imperativa la implementación de una política pública de planeación integral, incluyente, participativa y sostenible, capaz de satisfacer las necesidades de la población y, al mismo tiempo, de dotar de la infraestructura y servicios adecuados para acrecentar aquellas economías de urbanización que promueven la competitividad de las ciudades a nivel internacional.

\section{El hábitat en la periferia metropolitana: Zumpango}

La Ciudad de México tendrá en 2020 una extensión de $3.140 \mathrm{~km}^{2}$, lo que representa $1.502 \mathrm{~km}^{2}$ de nuevo suelo urbano, casi duplicando su extensión actual. El municipio periférico de Zumpango está en este constante proceso de urbanización, pues desde 2004 se ha intensificado la construcción de nuevos conjuntos habitacionales, y en el periodo 2000-2010 ha atraído a más de 60.000 nuevos residentes, lo que se refleja en 16.730 nuevos hogares y más de 18.000 viviendas (INEGI, 2010).

La periferia de la capital mexicana se caracteriza por un patrón de urbanización disperso. Como en todas las grandes urbes latinoamericanas, se observa una mayor densidad de población en el área central, hasta llegar a una dispersión extrema en los municipios periféricos. El municipio de Zumpango se encuentra entre las diez demarcaciones con más baja densidad urbana, lo que describe su patrón de urbanización altamente extendido con una gran cantidad de baldíos. Además, la ciudad tiene una baja intensidad de uso de suelo, pues solamente $20,8 \%$ de sus superficie es utilizada. ${ }^{3}$ En lo particular, Zumpango tiene una proporción inferior

Esta relación no necesariamente es mala, pues se tienen áreas naturales de conservación ecológica en la mayoría de las delegaciones periféricas del Distrito Federal y de algunos municipios conurbados. 
como resultado de su localización periférica y mezcla con actividades rurales. En ese sentido, ante los nuevos patrones de utilización del suelo, es probable que los futuros conjuntos habitacionales utilicen más las áreas rurales que los baldíos en los intersticios de la actual área urbana, lo que reproduce la tendencia hacia la dispersión. De esta manera, se configura un patrón de urbanización basado en núcleos de población relativamente aislados entre sí, constituidos por conjuntos habitacionales. Este esquema dificulta la articulación metropolitana y constituye un obstáculo para la generación de los espacios de vida, ${ }^{4}$ ante la falta de espacios de convivencia social.

\section{MAPA I | Área metropolitana de la Ciudad de México}

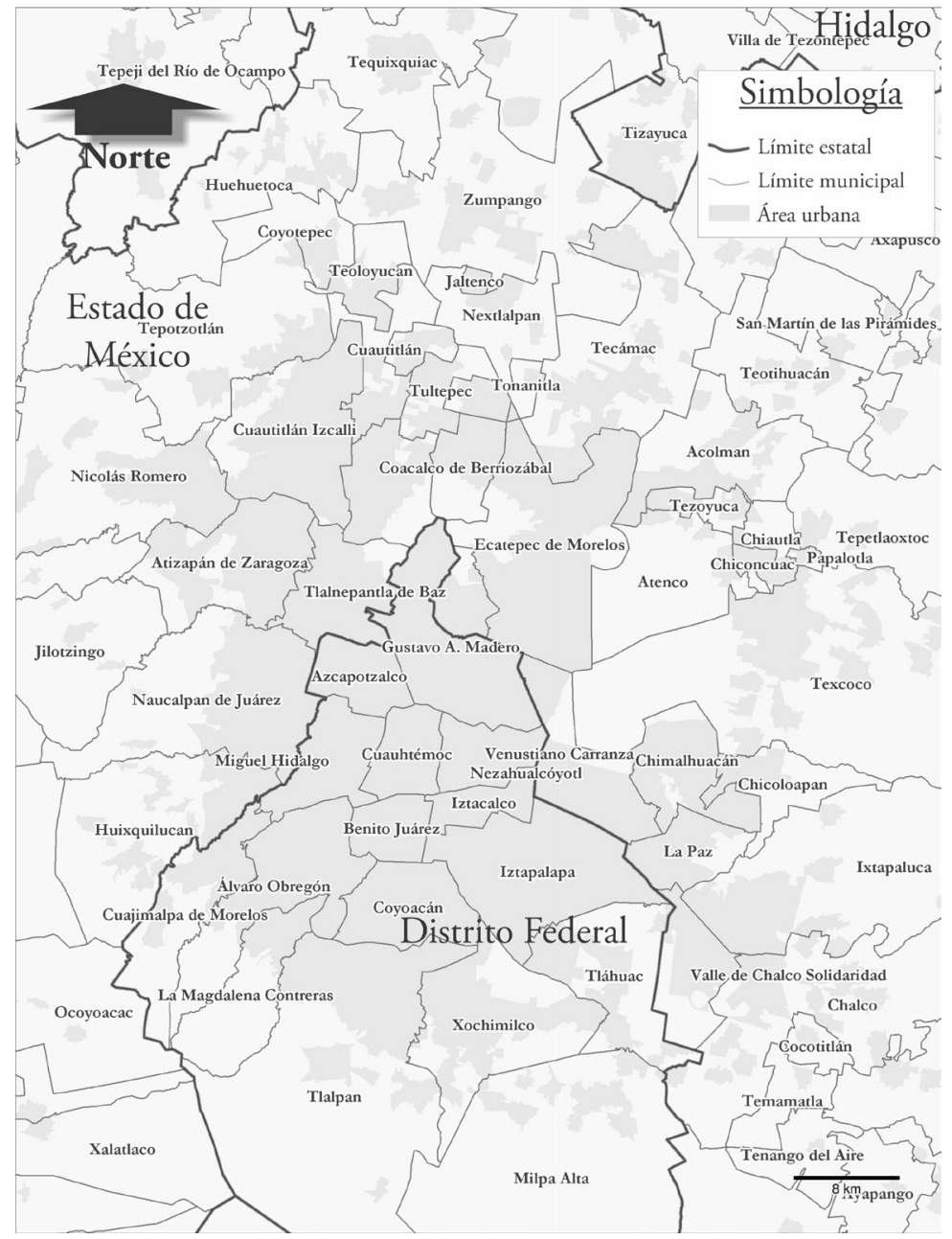

FUENTE ELABORACIÓN PROPIA A PARTIR DEL MARCO GEOESTADÍSTICO NACIONAL (INEGI, 2OIO)

4 El espacio de vida ofrece la posibilidad de considerar algunos desplazamientos temporales. En fin, una extensión del concepto de movilidad personal a unidades sociales más complejas, como la familia o la comunidad (Courgeau, 1988, p. 878). 
Durante el periodo 2000-2010 se autorizó la construcción de 338 conjuntos habitacionales en los municipios del Estado de México. Estos representan cerca de 635.000 viviendas que albergan 2,9 millones de personas. Los municipios que mayor población captan por motivos de ocupación residencial son Tecámac, Zumpango, Huehuetoca y Chalco. El municipio de Zumpango experimentó mayor grado de urbanización a partir del auge inmobiliario que comenzó en 2004 con dos conjuntos habitacionales. En el periodo de 2008 a 2010 se registró la construcción de 16 más, que representan 365.000 nuevos habitantes en tres años. Con ello, en más de 3.158 hectáreas de tierra destinada a la agricultura de riego con alta productividad se construyen masivos fraccionamientos de interés social (Eibenschutz, 2006, p. 35).

Gran parte de los nuevos residentes de Zumpango han emigrado originalmente de las áreas centrales e intermedias de la misma Ciudad de México, es decir, existe un fenómeno de expansión urbana por el crecimiento natural de la urbe y la generación de nuevos hogares. Según la Encuesta Movilidad Residencial en Tecámac y Zumpango, Estado de México (entidad a la que pertenece Zumpango), de la Ciudad de México provienen 254 residentes (62,9\%) y del Distrito Federal, entidad que ejerce la mayor centralidad metropolitana, 140 personas (34,6\%). El 67\% de ellos arribó a Zumpango entre 2008-2012 (Isunza, 2013, p. 7).

\section{Deficiente hábitat limita el habitar}

Para caracterizar el entorno de la vivienda y evaluar el hábitat, se requiere conocer la existencia de servicios públicos, y también la percepción de los habitantes. En esta sección se evalúa las condiciones del hábitat a partir del inventario existente de servicios de infraestructura y de equipamiento, ${ }^{5}$ disponibilidad de áreas abiertas de convivencia, ${ }^{6} \mathrm{y}$ condiciones de accesibilidad. Ciertamente, las personas construyen sus hábitats y los viven con diversas vulnerabilidades, carencias y desequilibrios, afectados por las dinámicas económicas, sociales, culturales, físico-espaciales y ambientales (Jiménez, 2013, p. 204), por lo que se espera que se identifiquen déficits en los diferentes elementos que se definieron para evaluar el hábitat en el municipio. Sin embargo, históricamente, la población ha sido capaz de adecuar su entorno y adaptarse a las limitantes urbanas, hasta apropiarse del espacio y construir un territorio habitable.

Existen estudios de caso donde se ha observado que la población residente de los nuevos conjuntos inmobiliarios modifica las condiciones físicas de sus viviendas en función de sus necesidades familiares, o instauran tiendas de abarrotes para mitigar la ausencia del servicio de abasto y tener una fuente de ingreso. No obstante, la adaptación de su hábitat urbano se hace más compleja ante la falta de otros elementos básicos, como clínicas, escuelas, iglesias, espacios abiertos, etcétera (Maya et al., 2013, p. 21).

5 Para el análisis de la dotación de redes de infraestructura se revisó principalmente el suministro de agua potable, drenaje, energía eléctrica y alumbrado público. En los elementos de equipamiento se limitó a estudiar los tres fundamentales: educación, salud y abasto.

6 En espacios abiertos se engloban las áreas verdes, como parques y jardines, así como la existencia de plazas y plazoletas; espacios destinados a la recreación y convivencia comunitaria. 
El primer elemento que se analizará es la red de servicios, conformada por el suministro de agua potable, sistema sanitario y red de electrificación. Según datos del Censo de Población (Inegi, 2010), los servicios básicos de agua, energía eléctrica y drenaje están cubiertos en $94,3 \%, 98,0 \%$ y 96,7\%, respectivamente. Esto se entiende, pues los conjuntos habitacionales se construyen ya con la dotación de estos servicios básicos, aspecto que es favorable en comparación con el proceso de urbanización de mediados del siglo xx. Hasta ese entonces, en los asentamientos informales las viviendas se caracterizaban normalmente por la irregularidad de su tenencia, ante lo cual el gobierno no las dotaba de servicios hasta después de un largo y tortuoso proceso de negociación.

Atendiendo a los resultados de la Encuesta Movilidad Residencial en Zumpango (Isunza, 2013), en la percepción de la población sobre el servicio de agua potable este aparece en términos generales como bueno, con un promedio de 8,6 (en la escala de 1 a 10), a la vez que los encuestados perciben una tendencia hacia la mejora en relación con su domicilio anterior. En situación similar se percibe el servicio de drenaje, aunque con una calificación de 7,8. Destacan conjuntos habitacionales como La Trinidad III y Villas de la Laguna, donde la calidad del servicio no representó una mejoría en cuanto a los lugares de residencia previos. Por último, la calidad en el alumbrado público es considerada regular, con un servicio definitivamente peor que en las zonas donde vivía previamente la población encuestada (cuadro 1).

CUAdro I | Zumpango: Percepción de la calidad ${ }^{a}$ de servicios de infraestructura, 2012

\begin{tabular}{|l|c|c|c|c|c|c|}
\hline \multirow{2}{*}{\begin{tabular}{l}
\multirow{2}{*}{$\begin{array}{l}\text { UANIDAD } \\
\text { HABITACIONAL }\end{array}$} \\
\cline { 2 - 7 }
\end{tabular}} & \multicolumn{2}{|c|}{ SERVICIOS DE AGUA } & \multicolumn{2}{c|}{ SERVICIO DE DRENAJE } & \multicolumn{2}{c|}{ ALUMBRADO PÚBLICO } \\
\hline ACTUAL & 8,6 & 7,1 & 7,8 & 7,9 & 6,7 & 8,3 \\
\hline $\begin{array}{l}\text { Total } \\
\text { Los Sauces }\end{array}$ & 8,9 & 7,0 & 8,3 & 7,8 & 7,5 & 8,7 \\
\hline $\begin{array}{l}\text { Arbolada, } \\
\text { Los Sauces II }\end{array}$ & 8,9 & 7,0 & 8,6 & 7,5 & 6,7 & 8,3 \\
\hline La Trinidad & 9,2 & 6,6 & 8,7 & 7,4 & 6,5 & 8,2 \\
\hline La Trinidad III & 8,6 & 6,9 & 7,6 & 7,6 & 5,3 & 8,0 \\
\hline $\begin{array}{l}\text { Nueva Villas de } \\
\text { la Laguna }\end{array}$ & 8,0 & 8,0 & 8,0 & 8,3 & 7,3 & 8,3 \\
\hline Santa Fe & 8,1 & 7,2 & 5,8 & 8,6 & 7,5 & 8,7 \\
\hline
\end{tabular}

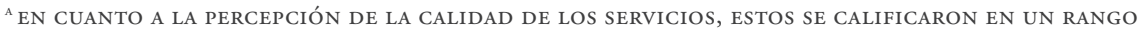
DE I A IO, REPRESENTANDO I UNA CALIDAD PÉSIMA Y IO UN EXCELENTE SERVICIO

FUENTE ELABORACIÓN PROPIA A PARTIR DE LOS RESUlTADOS DE LA ENCUESTA LEVANTADA A LA POBLACIÓN DE ZUMPANGO (ISUNZA, 2OI3)

Indudablemente, la ventaja de la construcción de conjuntos habitacionales en Zumpango es la dotación de servicios de infraestructura, donde la calidad del suministro del agua potable es buena, pero con un servicio de drenaje y alumbrado 
público regular. No ocurre lo mismo respecto de la dotación de otros elementos fundamentales, como el equipamiento urbano, del cual se analizará principalmente el sector educativo, de salud y de abasto.

Cuadro 2 |Zumpango: Inventario de instituciones educativas y población en edad de estudiar, 2010

\begin{tabular}{|l|c|c|c|c|c|}
\cline { 2 - 6 } \multicolumn{1}{c|}{} & TOTAL & PRIMARIA & SECUNDARIA & $\begin{array}{c}\text { MEDIA } \\
\text { SUPERIOR }\end{array}$ & SUPERIOR \\
\hline Escuelas & 194 & 67 & 70 & 18 & 4 \\
\hline Alumnos & 46.752 & 7.771 & 23.748 & 3.847 & 2.886 \\
\hline $\begin{array}{l}\text { Potenciales } \\
\text { usuarios }\end{array}$ & 61.977 & 6.978 & 23.589 & 8.581 & 13.695 \\
\hline
\end{tabular}

FUENTE ELABORACIÓN PROPIA A PARTIR DE DIRECCIÓN GENERAL DE PLANEACIÓN, PROGRAMACIÓN Y ESTADÍSTICAS EDUCATIVAS (2OI4) E INEGI, 2010

A escala municipal, la dotación de instituciones educativas tiene una cobertura adecuada en los primeros niveles de enseñanza, pues atiende a más población que la potencial demanda en el municipio. Sin embargo, la oferta educativa del nivel medio superior y superior no cubre la totalidad de la población en su respectivo rango de edad de asistencia. ${ }^{7}$ Parte de este déficit se puede justificar por el grado de deserción y abandono de estudios de la población, el cual se incrementa en los niveles de escolaridad de secundaria en adelante. ${ }^{8}$ Por lo tanto, el grado de dotación de servicios educativos es parcialmente adecuado en función de la cantidad de población residente (cuadro 2).

CUADRO 3 | Zumpango: inventario y demanda de servicio de salud, 2013

\begin{tabular}{|l|c|c|c|}
\hline \multirow{2}{*}{\multicolumn{1}{|c|}{ NIVEL DE SERVICIO }} & \multirow{2}{*}{ UNIDADES } & \multicolumn{2}{c|}{ SUPERFICIE CONSTRUIDA } \\
\cline { 3 - 4 } & & EXISTENTE & REQUERIDA \\
\hline Total & 19 & 39.094 & 22.006 \\
\hline $\begin{array}{l}\text { Unidades médicas de primer } \\
\text { contacto }\end{array}$ & 12 & 3.950 & 7.912 \\
\hline Hospitales & 3 & 16.565 & 14.094 \\
\hline
\end{tabular}

FUENTE ELABORACIÓN PROPIA A PARTIR DE LOS DATOS OTORGADOS POR LA DIRECCIÓN GENERAL DE INFORMACIÓN EN SALUD (2OI4), POR EL INSTITUTO DE ADMINISTRACIÓN Y AVALÚO DE BIENES NACIONALES (INDAABIN (2OI2) y LA DIRECCIÓN GENERAL DE INFRAESTRUCTURA Y EQUIPAMIENTO (I999)

7 La estimación de la población potencialmente usuaria de los servicios educativos se estableció a partir de rangos de edad: 4-5 ańos cumplidos, preescolar; 6-12 años, primaria; 13-15 ańos, secundaria; 16-18 años, media superior; y 19-23 años, superior.

8 A nivel nacional, $94 \%$ de la población infantil asiste a la educación básica; sin embargo, para la secundaria, solo $57 \%$ de los adolescentes acude al escuela, y de la población de 20 ańos y más, un reducido 5\% continúa sus estudios a nivel superior (INEGI, 2010). 
En el tema de los servicios de salud se tiene un importante déficit en la dotación de clínicas de primer contacto, mientras que para los hospitales se superan los requerimientos de superficie construida (cuadro 3). La carencia, que casi duplica la existencia en el servicio de salud de primer contacto, representa un riesgo para la población que no tiene la infraestructura suficiente para contener las enfermedades en sus etapas iniciales. El adecuado abastecimiento de estos establecimientos es vital, pues ellos representan el primer contacto de las personas con el servicio de salud y es donde se realizan las acciones de promoción, prevención, curación y rehabilitación de enfermedades. La ausencia de este servicio o su deficiencia comportan una alta vulnerabilidad para la salud pública, pues en caso de enfermedad, ésta puede escalar a etapas más avanzadas que requerirían hospitalización y un incremento del gasto familiar.

Por último, se observa una gran proliferación de pequeñas tiendas de abarrotes en el municipio. El Directorio Estadístico Nacional de Unidades Económicas (DENUE) registró 1.411 establecimientos, que en su gran mayoría son autogenerados por la misma población ante la ausencia de mercados públicos formales. Solamente se identificó un mercado público, localizado en Zumpango de Ocampo, la cabecera municipal. Además, se ubicaron 11 tiendas de autoservicio, la mayoría en los asentamientos ya existentes previos a la construcción masiva de los conjuntos habitacionales (INEGI, 2012b).

Si bien es cierto que el abastecimiento de los elementos de equipamiento está medianamente cubierto, a excepción del servicio de salud de primer nivel y de educación media superior y superior, su emplazamiento se encuentra fuera de los extensos fraccionamientos. Mediante la localización de los establecimientos de educación, salud y abasto con el DENUE, se observó que ellos tienden a ubicarse fuera de los conjuntos habitacionales. A excepción de La Trinidad, en el resto de los fraccionamiento se observa una clara ausencia de infraestructura educativa, de salud y de abasto (mapa 2).

Desde el punto de vista de la distribución espacial, el actual equipamiento tiende a localizarse en los asentamientos originales previos a la intensa construcción de desarrollos inmobiliarios, de tal forma que estas nuevas urbanizaciones, al no tener prevista la dotación de servicios básicos, fuerzan a sus habitantes a satisfacer sus necesidades básicas de consumo fuera de sus barrios. Esto representa la construcción de un hábitat incompleto, donde el déficit de los elementos de equipamiento dificulta la generación de espacios de vida logrados a través de actividades como ir a la escuela, de compras o a las clínicas de salud. Para los residentes, la ausencia de estos servicios degrada el entorno urbano como mera zona de transición entre sus actividades urbanas y sus espacios de vivienda, en oposición a las posibilidades de construcción de la identidad y cohesión social.

Por otro lado, el municipio dispone de una gran cantidad de áreas libres, consecuencia de los numerosos baldíos urbanos ubicados tanto en los asentamientos originarios como en los alrededores de las nuevas unidades habitacionales, que en lugar de ubicarse contiguamente a las áreas urbanas preexistentes, se emplazan en sectores totalmente aislados. Cabe resaltar que estos baldíos o vacíos urbanos no representan un espacio propicio para las actividades de convivencia, pues son propiedad privada, por lo que está restringido su acceso y no han sido adaptados por la población 
para la realización de sus actividades comunitarias. En el caso de los conjuntos habitacionales, las áreas verdes son en su mayoría zonas residuales, consecuencia del diseño deficiente de la lotificación. Por lo general se encuentran sobre las avenidas principales, lo que representa un riesgo para las actividades infantiles, o se vuelven espacios con alta incidencia de los ruidos provenientes del paso de vehículos.

MAPA 2 Zumpango: distribución del equipamiento urbano de educación, salud y abasto, 2012

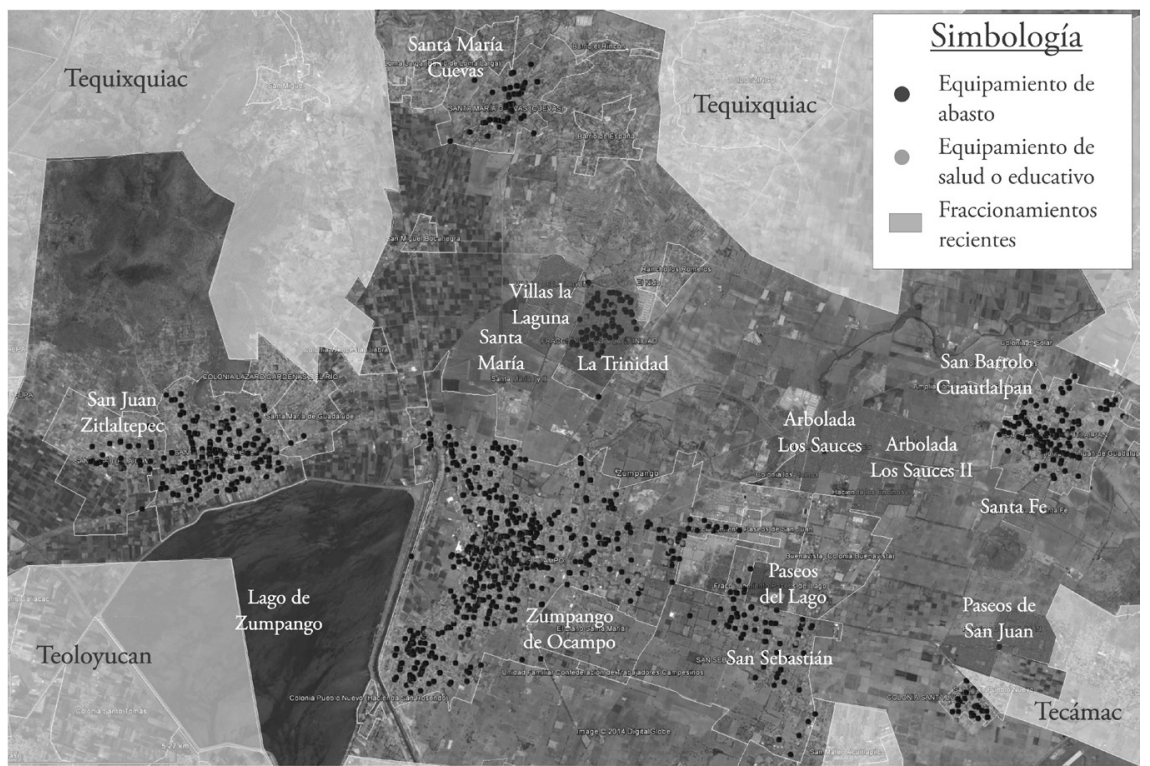

FUENTE ELABORACIÓN PROPIA A PARTIR DEL DIRECTORIO ESTADÍSTICO NACIONAL DE UNIDADES ECONÓMICAS, INEGI

La transformación de espacios baldíos en parques, jardines y áreas de juego fortalecería la función del espacio público otorgando mayor habitabilidad a los asentamientos, en el sentido de apropiación y territorialización de aquellos espacios a los que se les asigna un simbolismo de recreo e interacción vecinal (Hernández, 2008, p. 112). Cabe mencionar que los espacios públicos son lugares de convivencia e interacción social por excelencia y constituyen los ejes articuladores de las ciudades y los servicios de equipamiento. Desempeñan también funciones sociales, ambientales, de movilidad y recreación, pero en la zona en estudio ni el déficit ni la inequidad en el acceso a espacios públicos han encontrado solución en la generación de espacios formales. Como consecuencia de la débil regulación del mercado de suelo, las cesiones de suelo para espacio público en el marco de proyectos habitacionales no son adecuadas, ni en cantidad, ni en calidad o localización.

En relación con la accesibilidad, se considera que los desplazamientos cotidianos constituyen determinantes claves de la organización y de la construcción de territorios, por lo que el estudio de movilidad espacial permite profundizar en el conocimiento de aquellas transformaciones territoriales que cobran mayor relevancia 
en el presente siglo, especialmente en las metrópolis a escala local. La movilidad cotidiana se identifica con las prácticas habituales y reiteradas de desplazamientos de corta duración y distancia vinculadas a distintos fines, donde estadísticamente predominan los desplazamientos al trabajo y al lugar de estudio, las compras y el ocio (Casado, 2008).

La construcción de un hábitat incluye el derecho de las personas a la movilidad, relacionada con la infraestructura física de la ciudad y de los entornos locales. Cuando se organiza la traza urbana y los sistemas de transporte para facilitar la movilidad de la población, esta adquiere la capacidad de acceder a los diferentes destinos con una baja inversión de tiempo y dinero. Un sistema vial y de transporte suficientemente articulado incrementa la interacción en la población, fortaleciendo tanto los lazos comunitarios al interior de los municipios como la realización del derecho a la ciudad. En este sentido, la accesibilidad es un atributo de la habitabilidad, porque se relaciona con la provisión de las condiciones necesarias para hacer posible la construcción de espacios de vida y de un espacio local polifuncional, que incluye el empleo, equipamiento (educativo, de salud, comercial, cultural, deportivo) y espacios públicos como elemento de cohesión social; también revela la dotación de infraestructura vial y de transporte, que permite la articulación local a la dinámica metropolitana. Por lo tanto, la accesibilidad física es trascendental en los procesos de vivir y habitar.

En Zumpango se observaron graves problemas de accesibilidad: la cobertura y calidad del servicio de transporte es insuficiente; no hay adecuada conectividad local ni metropolitana; la red formal de transporte público en lo que respecta a las rutas, número y tipo de unidades, confort y seguridad, no se corresponde con la demanda de los residentes; además, el costo de transporte es elevado en relación con los ingresos medios de la población. Todo ello ha propiciado la operación de redes locales de transporte informal. Cuando los tiempos y costos de desplazamiento se incrementan, los espacios de vida se reducen a un contexto cada vez más limitado. La ciudad es incapaz de ser habitada mediante los desplazamientos peatonales $\mathrm{u}$ otros modos de transporte de baja velocidad, como puede ser la bicicleta. Por ello, se vuelve una zona de transición hacia destinos muy focalizados, como pueden ser los centros de empleo, educativos, recreativos o culturales.

Analicemos el tema de la movilidad. La movilidad denota el acceso a la ciudad, a sus territorios, a todos los tipos de oportunidades que la ciudad ofrece, incluyendo los vínculos sociales, de tal suerte que trabajar, consumir o relacionarse con los otros implica necesariamente desplazarse (Avellaneda \& Lazo, 2011, p. 49).

Respecto al modo de desplazamiento, la encuesta levantada reveló que, en Zumpango, el automóvil privado no es tan utilizado como el transporte público, y los desplazamiento caminando tienen relevancia. Las distancias a las zonas que ejercen centralidad son largas.

La estructura territorial de Zumpango tiene un alto grado de desarticulación interna, debido a que las unidades habitacionales configuran núcleos de población relativamente aislados entre sí, lo que obstaculiza la cohesión social de los nuevos residentes y dificulta en gran medida la movilidad. La situación se agrava por el incremento en la demanda de unidades de transporte público de mediana 
velocidad, por el aumento del automóvil privado, así como por la inexistencia en Zumpango de una red de transporte con los atributos necesarios de funcionalidad, seguridad, confort y cobertura, según lo revelan los hallazgos de la encuesta. De las 297 personas que se desplazan en transporte público, 6\% indicó emplear en ello de 30 minutos a una hora (sumando el tiempo empleado de ida y vuelta); 18\% requiere de 1 a 2 horas; $13 \%$ hasta 3 horas, y 25\% aseguró emplear diariamente 4 horas o más en desplazarse. Es decir, el tiempo de traslado en transporte público es elevado debido a la dispersión de los asentamientos humanos, donde incluso las unidades habitacionales están muy lejanas entre sí. Nuevamente se manifiesta una contradicción entre el habitar y el hábitat.

El excesivo tiempo y costo implicados en las personas que diariamente son obligadas a salir de sus municipios, tiene graves implicaciones sobre su calidad de vida, además de la insuficiencia de la cobertura y calidad del transporte público y la poca o nula conectividad de los desarrollos inmobiliarios, ponen en evidencia la ausencia de un vínculo de la movilidad con el ordenamiento territorial. (Isunza, 2015, p. 234)

El impacto de la distancia, el uso social del tiempo y la escasa accesibilidad en el entorno municipal, terminan minando la capacidad de las personas para desplazarse y construir su espacio de vida; es justamente esta una importante causa del abandono de la vivienda por parte de familias que adquirieron un crédito hipotecario para tener acceso a ella, pero que se enfrentan a la escasa habitabilidad del asentamiento.

\section{El abandono de la vivienda}

El tema de la vivienda deshabitada refleja claramente la baja calidad del hábitat $\mathrm{y}$, como consecuencia, la incapacidad de habitar los nuevos conjuntos habitacionales. Los territorios morfológicamente desmembrados, fracturados, separados y divididos, fragmentan las condiciones espaciales y temporales de la vida cotidiana de sus habitantes (Jirón \& Mansilla, 2014, p. 13). El aislamiento físico, aunado a la deficiente dotación de servicios y al inadecuado sistema de transporte, ocasiona una segregación que debe ser cubierta por una alta inversión de tiempo y dinero en los traslados de la población. De esta forma, la población residente de los conjuntos habitacionales de Zumpango se ve obligada a realizar gran parte de sus actividades cotidianas muy lejos de su domicilio. Como resultado de estas graves carencias en los servicios de equipamiento, áreas públicas y accesibilidad metropolitana, un número creciente de familias ha decidido abandonar sus viviendas, empujadas por la necesidad de construir un ambiente integrado a su vida cotidiana.

En 2005, en Zumpango existían 3837 viviendas deshabitadas, ${ }^{9}$ lo que representaba apenas $11,9 \%$ del total de las viviendas, mientras que 1.331 se destinaban a uso

Se entiende por vivienda deshabitada a la "vivienda particular que está completamente construida y disponible para ser habitada y al momento del levantamiento censal no tiene residentes habituales, no es de uso temporal y no es utilizada como local en actividad económica" (INEGI, 2011, p. 56). 
temporal ${ }^{10}$ lo que corresponde a 4,1\% (INEgi, 2005). Para 2010, la vivienda deshabitada se incrementó drásticamente a 29.345, lo que elevó su porcentaje a 39,7. Este grado de desocupación solo estuvo por debajo del de Huehuetoca, otro municipio urbano periférico, donde $44,7 \%$ de sus viviendas se registraron deshabitadas. Además, la vivienda de uso temporal tuvo un ligero incremento, al contabilizarse 6.948 unidades, lo que significó 9,4\% (INEGI, 2010).

Importante proporción de las viviendas deshabitadas está vinculada con el altísimo grado de desocupación de los conjuntos habitacionales. En seis conjuntos habitacionales ubicados en el municipio, se cuantificaron 43.585 viviendas, de las cuales $71 \%$ se registró como deshabitadas en 2012. El caso más drástico es Villas de la Laguna, donde $77,5 \%$ de las viviendas no están habitadas. Esto se entiende, pues además de las carencias de equipamiento, espacios públicos y la deficiente accesibilidad de la zona, la población encuestada evaluó la calidad de los servicios de infraestructura de estos fraccionamientos como peor en referencia al domicilio previo (cuadro 4).

CUADRo 4 | Zumpango: grado de ocupación de las viviendas de los conjuntos habitacionales, 2012

\begin{tabular}{|l|c|c|c|c|c|}
\hline \multicolumn{1}{|c|}{$\begin{array}{c}\text { UNIDAD } \\
\text { HABITACIONAL }\end{array}$} & TOTALES & HABITADAS & $\%$ & DESHABITADAS & $\%$ \\
\hline Total & 43.585 & 12.636 & 29,0 & 30.949 & 71,0 \\
\hline Villas de la Laguna & 5.146 & 1.160 & 22,5 & 3.986 & 77,5 \\
\hline La Trinidad & 8.746 & 2.943 & 33,6 & 5.803 & 66,4 \\
\hline Paseos de San Juan & 9.459 & 2.996 & 31,7 & 6.463 & 68,3 \\
\hline Paseos del Lago & 2.707 & 937 & 34,6 & 1.770 & 65,4 \\
\hline Arbolada Los Sauces & 10.347 & 2.781 & 26,9 & 7.566 & 73,1 \\
\hline Arbolada Los Sauces II & 7.180 & 1.819 & 25,3 & 5.361 & 74,7 \\
\hline
\end{tabular}

FUENTE INEGI (2OI $2 \mathrm{~A})$

En contraste, si bien es cierto que las unidades Paseos del Lago y La Trinidad tienen un grado de abandono por encima del $50 \%$, son las que registraron menos proporción de viviendas deshabitadas. En el caso de La Trinidad, se puede explicar porque en su interior existen servicios de equipamiento, y aunque habría que revisar su calidad, los habitantes tienen mayor accesibilidad a estos servicios sin necesidad de trasladarse a la cabecera municipal. Paseos de Lago está adjunta al poblado de San Sebastián, y dado que el fraccionamiento no es tan grande, la cercanía de los servicios del poblado los hace accesibles (mapa 2).

Debido a la continua expansión del АMCM, los conjuntos habitacionales de interés social se encuentran cada vez más alejados de los centros de empleo, lo que

10 La vivienda temporal se considera aquella "vivienda particular que está totalmente construida y disponible para ser habitada y que al momento del levantamiento censal está destinada a vacacionar, descansar o vivir algunos días, semanas o meses, no tiene residentes habituales ni se ocupa como local con actividad económica" (INEGI, 2011, p. 56). 
incrementa a un grado incosteable el traslado de los residentes entre sus domicilios y sus destinos laborales, de estudio, compras u otros. Otro elemento necesario de considerar es que la mayor parte de la población residente ha dejado de estar compuesta por familias inmigrantes de las áreas rurales, para provenir predominantemente de las áreas centrales y semiperiféricas de la megaurbe. Mientras que la población inmigrante campesina atravesaba por un proceso de cambio total de sus hábitos, cotidianidad y espacios de vida, la nueva población residente conserva sus empleos en la metrópoli y algunos espacios de vida, como resultado de sus vínculos con la población que vive en la ciudad, familiares o amigos. Sin embargo, pese a conservar sus hábitos de realizar sus actividades en la urbe, ante el aislamiento de su nueva vivienda deciden abandonarla para buscar aproximarse a los espacios que frecuentemente habitan, para reducir los excesivos costos de transporte; es decir, no internalizan los altos costos, el tiempo y el esfuerzo implicado en su desplazamiento.

\section{Conclusiones: procesos de apropiación del espacio urbano}

En las actuales periferias metropolitanas coexisten los asentamientos históricamente construidos, ya sean formales o informales, con una fuerte presión hacia la expansión urbana por parte de las empresas inmobiliarias. Los que en tiempos pretéritos fueron espacios rururbanos, ahora están sometidos a cambios en su configuración territorial y en el perfil socioeconómico del habitante.

En esta transición, los caminos tradicionales de ocupación del suelo a partir de la autoconstrucción de la vivienda, y de construcción del espacio público a partir del apoyo gubernamental, permitían un proceso de apropiación, a partir del cual se generaba un hábitat conformado en función de las necesidades de la población residente. Ahora, con la nueva lógica de producción de los espacios mediante las empresas inmobiliarias, se ofrece un espacio 'terminado', con conjuntos unifamiliares o multifamiliares que no dan cabida a transformaciones ni al interior de la vivienda ni en el entorno. ${ }^{11}$ Existen pocos casos en que se ofrecen viviendas progresivas donde la constructora solamente entrega el pie de casa, y a los propietarios se les dan sugerencias de ampliación de su vivienda.

Respecto al diseño urbano, la zonificación ya está definida por algún uso, predominantemente habitacional, por lo que la inserción de espacios de uso público se hace prácticamente imposible. Esto dificulta el proceso de apropiación del espacio y, aunado a que los servicios de equipamiento y de áreas de convivencia, recreación y trabajo se encuentran fuera de los conjuntos habitacionales, tiende a radicalizar a una escala intramunicipal el fenómeno de zonas dormitorio. Es decir, a escala metropolitana, la periferia urbana ha sido el destino de la mayoría de las nuevas opciones de vivienda, sin una creación de centros de empleo.

En el interior de esta nueva periferia urbana es posible identificar masivos conjuntos habitacionales, sin las condiciones necesarias para crear un barrio donde la población pueda acceder a los servicios urbanos. Ello obliga a las personas a

11 En Staines (2013) se recogen varios estudios sobre la adaptación de las viviendas en conjuntos habitacionales en ciudades latinoamericanas. 
salir de dichos fraccionamientos para realizar sus actividades cotidianas, lo que termina por impedir la apropiación del espacio público en el contexto inmediato a la vivienda.

Junto a lo anterior como factores que obstaculizan la generación de un habitar, se tiene la condición del perfil sociocultural de la población, donde los habitantes originarios ubicados en la entonces área de influencia de la metrópoli mantenían usos y costumbres semiurbanos, con un mayor contacto con la naturaleza. Los nuevos pobladores, destinatarios de los grandes conjuntos habitacionales, son esencialmente habitantes de la metrópoli con una previa residencia en las áreas centrales o intermedias. Este segmento de la población, con costumbres eminentemente urbanas, se ve forzado a buscar vivienda propia en estos aislados fraccionamientos, única posibilidad a la que tiene acceso en el marco de la oferta de créditos hipotecarios y de vivienda de interés social. En este proceso, los nuevos residentes, cuyos espacios de vida previos se incrustaban en la dinámica metropolitana, se enfrentan al problema de construir espacios de vida en un nuevo contexto que no satisface sus necesidades básicas y se contrapone a la cotidianidad experimentada en su anterior vivienda. Ante la falta de servicios de equipamiento, deficiencias en la dotación de áreas verdes, la baja accesibilidad metropolitana de la nueva ubicación de su vivienda, la dificultad de adaptación del espacio privado y público a sus necesidades, mantienen sus estilos de vida previos o, en el mejor de los casos, acuden a los asentamientos originales del municipio para satisfacer sus necesidades de servicios urbanos.

La incapacidad de apropiarse de su nuevo entorno urbano, cuyo hábitat no satisface sus necesidades sociales, culturales y económicas, ocasiona que un importante segmento de personas abandone sus domicilios y retorne a los antiguos hábitos y espacios de vida. La construcción de un hábitat inadecuado genera problemas sociales en estos nuevos espacios, pues el abandono de las viviendas facilita la inseguridad y el robo en ellas, lo que degrada aún más la habitabilidad.

Si bien la construcción del espacio urbano se ha dejado bajo la responsabilidad de las empresas inmobiliarias, delegando en ellas funciones que tradicionalmente asumían las instituciones públicas, existen graves carencias en cuanto al diseño de las nuevas urbanizaciones, como resultado de la inexistencia de regulaciones adecuadas en cuanto a dos aspectos fundamentales:

a. Su emplazamiento. Las empresas deciden construir en zonas muy aisladas y desarticuladas de la urbe, con la finalidad de obtener bajo precio de suelo y así incrementar su rango de ganancia; y

b. Su diseño. No se prevén las necesidades de espacios públicos y de equipamiento. La reducción o supresión de estos espacios de donación a la actividad comunitaria son causados nuevamente por la búsqueda por parte de la empresa inmobiliaria en cuanto a incrementar la rentabilidad del proyecto, al aumentar el área vendible con más lotes y viviendas.

Respecto a la gestión del hábitat, se presenta una paradoja: los nuevos conjuntos habitacionales no atienden el rezago habitacional de la población local en Zumpango, mientras que los nuevos residentes no se adaptan del todo al nuevo 
entorno local; los gobiernos locales se ven rebasados, al no responder a la demanda creciente de servicios urbanos básicos y de condiciones de habitabilidad.

Considerando lo anterior, es fundamental la regulación de la construcción de los nuevos espacios urbanos, la planeación territorial local y la gestión por parte de las autoridades. Las características del espacio público y las actividades cotidianas que se realizan en él son determinantes para lograr un modelo de ciudad con las condiciones de vida necesarias para lograr una urbe más equitativa e incluyente. Adicionalmente, se requiere fortalecer la actividad económica local para incrementar la oferta de empleo y, con ello, reducir los desplazamientos de largas distancias.

Finalmente, el problema de la escasa habitabilidad deviene del esquema de urbanización basado en mayor dispersión de los espacios periurbanos, que lejos de atender el rezago habitacional, favorece la mercantilización de la ciudad y el protagonismo del capital inmobiliario.

\section{Referencias bibliográficas}

Arcas-Abella, J., Pagès-Ramon, A. \& Casals-Tres, M. (2011). El futuro del hábitat: repensando la habitabilidad desde la sostenibilidad. El caso español. Revista INVI, 26(72), 65-93. http://revistainvi.uchile.cl/index.php/INVI/article/view/556

Avellaneda, P. \& Lazo, A. (2011). Aproximación a la movilidad cotidiana en la periferia pobre de dos ciudades latinoamericanas. Los casos de Lima y Santiago de Chile. Revista Transporte y Territorio (4), 47-58. www.rtt.filo.uba.ar/RTT00404047.pdf

Brites, W. (2012). Las adversidades del hábitat en conjuntos habitacionales de población relocalizada. En T. Bolicar \& J. Erazo (Coords.), Dimensiones del hábitat popular latinoamericano (pp. 121-142). Quito: Facultad Latinoamericana de Ciencias Sociales (Flacso) / Consejo Latinoamericano de Ciencias Sociales (Clacso) / Instituto de la Ciudad.

Casado, J. M. (2008). Estudios sobre movilidad cotidiana en México, Scripta Nova. Revista Electrónica de Geografía y Ciencias Sociales, 12(273). http://www.ub.edu/geocrit/sn/ sn-273.htm

Courgeau, D. (1988). Méthodes de mesure de la mobilité spatiale: migrations internes, mobilité temporaire et navettes. Population, 43(4-5), 877-880. doi: 10.2307/1533496

De Mattos, C. (2015). Revolución urbana. Estado, mercado y capital en América Latina. Santiago, Chile: Ril Editores / Colección Estudios Urbanos Uc.

Dirección General de Información en Salud (2014). Cubos Dinámicos. México: Secretaría de Salud. http://www.dgis.salud.gob.mx/contenidos/basesdedatos/BD_Cubos.html

Dirección General de Infraestructura y Equipamiento (1999). Sistema Normativo de Equipamiento Urbano, Tomo II. México: Secretaría de Desarrollo Social.

Dirección General de Planeación, Programación y Estadística Educativa (2014). Sistema de Consulta Interactiva de Estadísticas Educativas. México: Secretaría de Educación Pública. http://www.snie.sep.gob.mx/estadisticas_educativas.html 
Eibenschutz, R. (2006). Estructura territorial. En Metropoli / 2025, Una visión para la Zona Metropolitana del Valle de México. Tomo II: Habitabilidad (pp. 23-58). México: Centro de Estudios para la Zona Metropolitana.

Hernández, G. \& Velázquez, S. (2014). Vivienda y calidad de vida. Medición del hábitat social en el México Occidental. Bitácora 24(1), 149-200. http://www.revistas.unal.edu.co/ index.php/bitacora/article/view/31463

Hurtado, H. \& Villagómez, J. (1992). Necesidades básicas y condiciones de vida. En Centro de Investigaciones Ciudad, Gestión del hábitat popular (pp. 37-39). Serie Ensayos Forhum, 2. Quito: Centro de Investigaciones Ciudad.

Instituto de Administración y Avalúo de Bienes Nacionales (Indaabin). Consulta en CD de los datos referentes al año 2012.

Instituto Nacional de Estadística y Geografía (INEGI). (2005). II Conteo de Población y Vivienda 2005. México: INEGI. http://www.inegi.org.mx/est/contenidos/proyectos/ccpv/cpv2005/

Instituto Nacional de Estadística y Geografía (INEGI). (2010). Censo de población y vivienda 2010. México: INEGI. http://www.inegi.org.mx/est/contenidos/proyectos/ccpv/cpv2010/

Instituto Nacional de Estadística y Geografía (INEGI). (2011). Sintesis metodológica y conceptual del Censo de Población y Vivienda 2010. Aguascalientes. México: inegi. http://www. inegi.org.mx/est/contenidos/espanol/metodologias/censos/sm_cpv2010.pdf

Instituto Nacional de Estadística y Geografía (INEGI). (2012a). Inventario Nacional de Vivienda. México: INEGI. http://www.beta.inegi.org.mx/app/mapa/inv/default.aspx

Instituto Nacional de Estadística y Geografía (INEGI). (2012b). Directorio Estadístico Nacional de Unidades Económicas. http://www.beta.inegi.org.mx/app/mapa/denue/

Isunza, G. (2013). Encuesta Movilidad Residencial en Tecámac y Zumpango, Estado de México. Informe del Proyecto Desarrollos Inmobiliarios y movilidad residencial en la ZMVM, registro 1470, Instituto Politécnico Nacional, octubre de 2013. http:// repositoriodigital.ipn.mx/handle/123456789/16878.

Isunza, G. (2015). La vivienda deshabitada en la periferia de la Ciudad de México. En S. Vidal (Coord.), Metrópolis en mutación (pp. 211-238). Buenos Aires: Café de las Ciudades.

Isunza, G. \& Méndez, B. (2011). Desarrollo inmobiliario y gobiernos locales en la periferia de la Ciudad de México. EURE, 37(111), 107-129. http://doi.org/10.4067/S025071612011000200005

Jiménez, W. (2013). Hábitat y vulnerabilidad, reflexiones desde lo conceptual. Revista Luna Azul, (37), 196-218. http://www.redalyc.org/articulo.oa?id=321729206013

Jirón, P. \& Mansilla, P. (2014). Las consecuencias del urbanismo fragmentador en la vida cotidiana de habitantes de la ciudad de Santiago de Chile. EURE, 40(121), 5-28. http://doi.org/10.4067/S0250-71612014000300001

Mac Donald, J. (2004). Pobreza y precariedad del hábitat en ciudades de América Latina y el Caribe. Serie Manuales, 38 [LC/L.22I4-P]. Santiago, Chile: Comisión Económica para América Latina y el Caribe (Cepal-onu). http://repositorio.cepal.org/bitstream/ handle/11362/5602/1/S0410827_es.pdf

Maya, E., Staines, E., Roux, R., Montalvo, S., Olivares, M., Herrera, R., Tapia, R., Larenas, J., Rengifo, C. \& Cuesta, A. (2013). Marco conceptual. Marco institucional. En E. Staines (Coord.), Habitabilidad y eficiencia energética en conjuntos habitacionales de interés social (pp. 17-65). Ciudad Juárez: Universidad Autónoma de Ciudad Juárez. 
Romero, G. \& Mesías, R. (2004). La participación en el diseño urbano y arquitectónico en la producción social de hábitat, México: Programa Iberoamericano de Ciencias y Tecnología para el Desarrollo.

Staines, E. (Coord.) (2013). Habitabilidad y eficiencia energética en conjuntos habitacionales de interés social. Ciudad Juárez: Universidad de Ciudad Juárez.

Tarchópulos, D. \& Ceballos, O. (2003). Calidad de la vivienda dirigida a los sectores de bajos ingresos en Bogotá, Bogotá: Centro Editorial Javeriano-Pontificia Universidad Javeriana.

Zulaica, L. \& Celemín, J. P. (2008). Análisis territorial de las condiciones de habitabilidad en el periurbano de la ciudad de Mar del Plata (Argentina), a partir de la construcción de un índice y de la aplicación de métodos de asociación espacial. Revista de Geografía Norte Grande, (41), 129-146. http://doi.org/10.4067/S0718-34022008000300007 